\title{
Prognostic genes of triple-negative breast cancer identified by weighted gene co-expression network analysis
}

\author{
LIGANG BAO $^{1}$, TING GUO $^{2}, \mathrm{JI}_{\mathrm{WANG}}^{3}, \mathrm{KAI}^{2} \mathrm{ZHANG}^{4}$ and $\mathrm{MAODE} \mathrm{BAO}^{5}$ \\ ${ }^{1}$ Emergency Department, Dongyang People's Hospital, Jinhua, Zhejiang 322100; \\ ${ }^{2}$ Department of Neurosurgery, Zhejiang Province Taizhou Hospital, Taizhou, Zhejiang 318000; \\ ${ }^{3}$ Department of Orthopaedics, 967th Hospital of The PLA Joint Logistics Support Force, Dalian, Liaoning 116021; \\ ${ }^{4}$ Department of Cardiology, Sir Run Run Shaw Hospital, Zhejiang University School of Medicine, Hangzhou, \\ Zhejiang 310016; ${ }^{5}$ Orthopedics Department, Dongyang Chinese Medicine Hospital, Jinhua, Zhejiang 322100, P.R. China
}

Received May 12, 2019; Accepted September 6, 2019

DOI: $10.3892 /$ ol.2019.11079

\begin{abstract}
Triple-negative breastcancer(TNBC) is characterized by a deficiency in the estrogen receptor (ER), progesterone receptor (PR) and HER2/neu genes. Patients with TNBC have an increased likelihood of distant recurrence and mortality, compared with patients with other subtypes of breast cancer. The current study aimed to identify novel biomarkers for TNBC. Weighted gene co-expression network analysis (WGCNA) was applied to construct gene co-expression networks; these were used to explore the correlation between mRNA profiles and clinical data, thus identifying the most significant co-expression network associated with the American Joint Committee on Cancer-TNM stage of TNBC. Using RNAseq datasets from The Cancer Genome Atlas, downloaded from the University of California, Santa Cruz, WGCNA identified 23 modules via $\mathrm{K}$-means clustering. The most significant module consisted of 248 genes, on which gene ontology analysis was subsequently performed. Differently Expressed Gene (DEG) analysis was then applied to determine the DEGs between normal and tumor tissues. A total of 42 genes were positioned in the overlap between DEGs and the most significant module. Following survival analysis, 5 genes [GIPC PDZ domain containing family member 1 (GIPC1), hes family bHLH transcription factor 6 (HES6), calmodulin-regulated spectrin-associated protein family member 3 (KIAA1543), myosin light chain kinase 2 (MYLK2) and peter pan homolog (PPAN)] were selected and their association with the American Joint Committee on Cancer-TNM diagnostic stage was investigated. The expression level of these genes in different pathological stages varied, but tended to increase in more advanced pathological stages.
\end{abstract}

Correspondence to: Dr Maode Bao, Orthopedics Department, Dongyang Chinese Medicine Hospital, 14 Wuning Eastern Road, Jinhua, Zhejiang 322100, P.R. China

E-mail: maode.bao123@gmail.com

Key words: triple-negative breast cancer, weighted gene co-expression network analysis, progression
The expression of these 5 genes exhibited accurate capacity for the identification of tumor and normal tissues via receiver operating characteristic curve analysis. High expression of GIPC1, HES6, KIAA1543, MYLK2 and PPAN resulted in poor overall survival (OS) in patients with TNBC. In conclusion, via unsupervised clustering methods, a co-expressed gene network with high inter-connectivity was constructed, and 5 genes were identified as biomarkers for TNBC.

\section{Introduction}

Triple-negative breast cancers (TNBCs) are characterized by the lack of HER2/neu, progesterone receptor (PR) and estrogen receptor (1) (ER) gene expression. The TNBC subtype constitutes $10-15 \%$ of total breast tumors and $80 \%$ of basal-like breast cancers (1). Triple-negative and basal-like tumors commonly have a high histological grade (1). TNBCs also have a poor prognosis and tend to result in earlier relapse compared with other subtypes of breast cancer (1). Additionally, TBNCs exhibit increased chemosensitivity compared with other genotypes of breast cancer (2), thus, chemotherapeutic methods are currently the most prevalently used medical treatments (3). These include epidermal growth factor receptor (EGFR)-targeted therapies, multi-tyrosine kinase inhibitors, poly-ADP ribose polymerase (PARP) inhibitors and anti-angiogenic agents (4). However, patients with TNBCs usually exhibit poorer outcomes following chemotherapy, compared with patients with breast cancers of other subtypes (3). Hence, it would be beneficial to identify novel biomarkers associated with the progression of TNBCs, and identify new targets to improve their precise diagnosis and treatment.

Weighted-correlation network analysis (WGCNA) has previously been performed to construct non-scale co-expressed gene networks (5-8). In the present study, WGCNA was performed to identify the hub-module that contained genes showing a strong correlation with the pathological stage of TNBC. Subsequently, differentially expressed gene (DEG) analysis was performed on RNAsequencing (RNAseq) data of TNBC. The hub-genes were defined as all genes contained in the overlap between the DEGs and the hub-module. Gene 


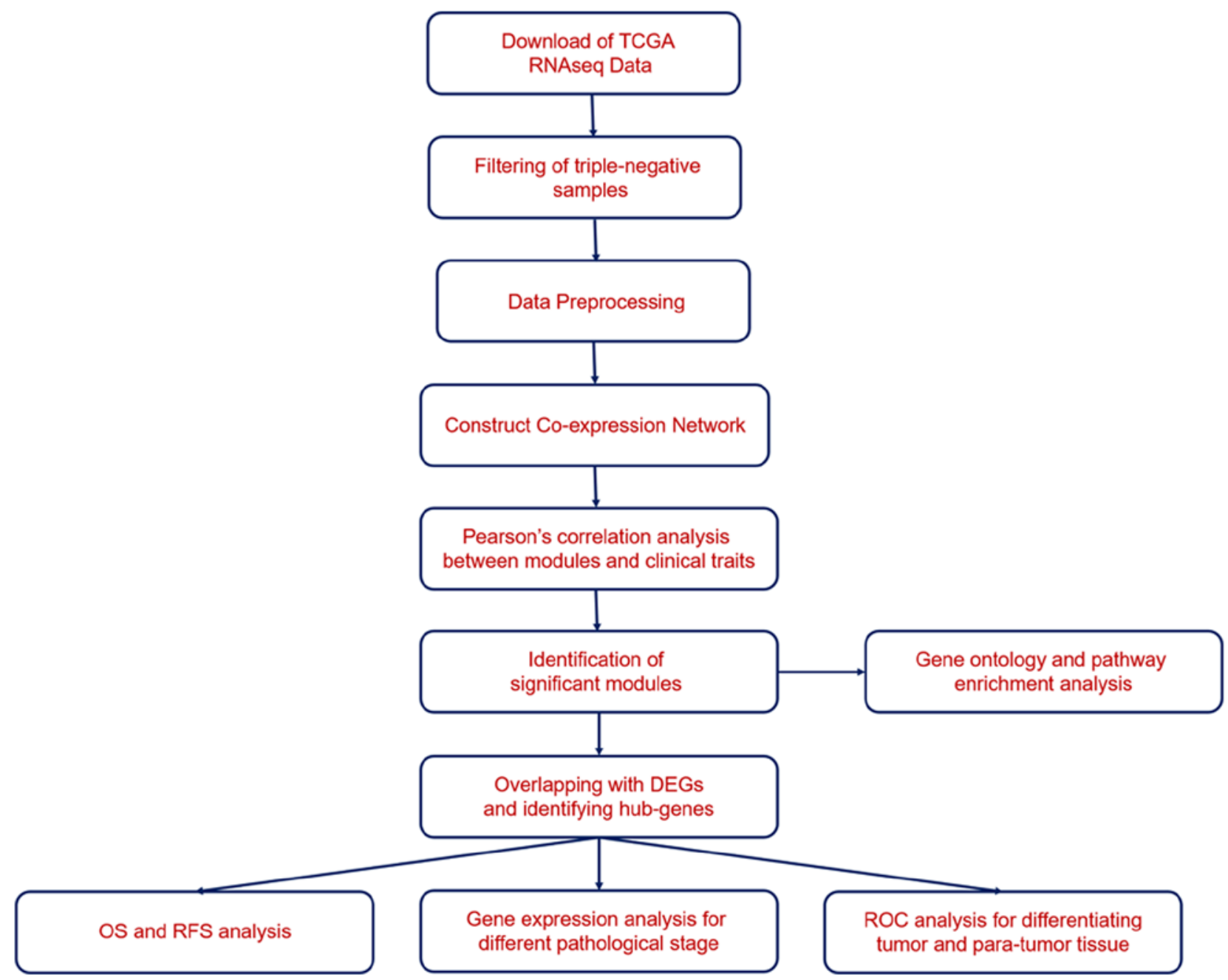

Figure 1. Workflow of the present study.

ontology (GO) and gene enrichment analyses were then employed, and identified several important terms in biological process, molecular function and cellular components. Survival analysis was also conducted, and 5 genes were selected from the hub-genes. Receiver operating characteristic (ROC) and Kaplan-Meier (KM) curves were plotted to indicate the capacity of these genes to differentiate tumor and para-tumor tissues, and to confirm the influence of these genes on the overall survival (OS) of patients with TNBC.

\section{Materials and methods}

Data processing and co-expression gene network construction using RNAseq data. A total of 1,240 RNAseq datasets from The Cancer Genome Atlas (TCGA) and clinical data for patients with breast cancer were downloaded from the University of California, Santa Cruz (UCSC) database using the Xena browser (https://xenabrowser.net/). The workflow is displayed in Fig. 1. The TNBC samples were filtered using the criteria of 'not expressing genes for ER, PR and HER2/neu'. $\mathrm{R}$ software (version 3.5.2; http://www.r-project.org) was applied to perform WGCNA analysis. WGCNA was used to construct the gene co-expression network; co-expression similarity $\left(S_{i, j}\right)$ was defined as the absolute value of the corre- lation coefficient between the mRNA expression profile of nodes $i$ and $j$ :

$$
s_{i, j}=\left|\operatorname{cor}\left(x_{i}, x_{j}\right)\right|
$$

Where $X_{i}$ and $X_{j}$ are mRNA expression values for genes $i$ and $j, S_{i, j}$ was calculated using the Pearson's correlation coefficient between genes $i$ and $j$. Weighted-network adjacency was defined by raising the co-expression similarity to a power:

$$
\alpha_{i, j}=\mathrm{s}_{i, j}^{\beta}
$$

$\beta \geq 1$. The power of $\beta=4$ and scale free $R^{2}=0.95$ were selected as the soft-thresholding parameters to ensure a signed scale-free gene network.

By evaluating the correlation between the pathological stage of TNBC and the module membership with the 'p. weighted', a high-correlated module was identified. The tan modules which had the most significant adjusted P-values were selected. Genes involved in the tan modules were presented using Cytoscape v3.4.0 (https://cytoscape.org). The genes in the tan module were selected as the input for $\mathrm{GO}$ and KEGG analysis, which was performed using Metascape (http://metascape.org/gp/index.html). 
A

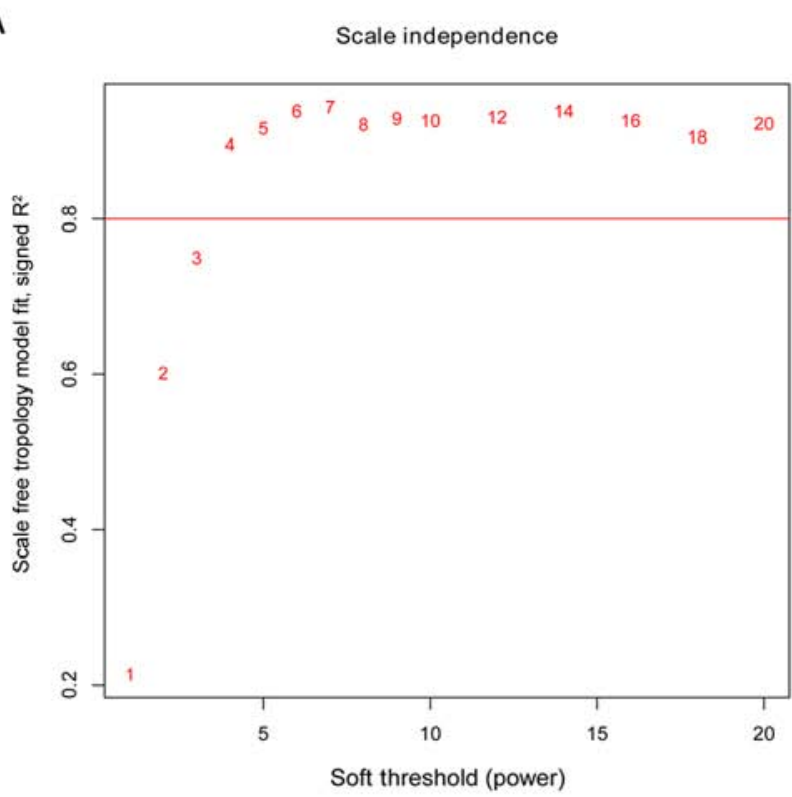

B

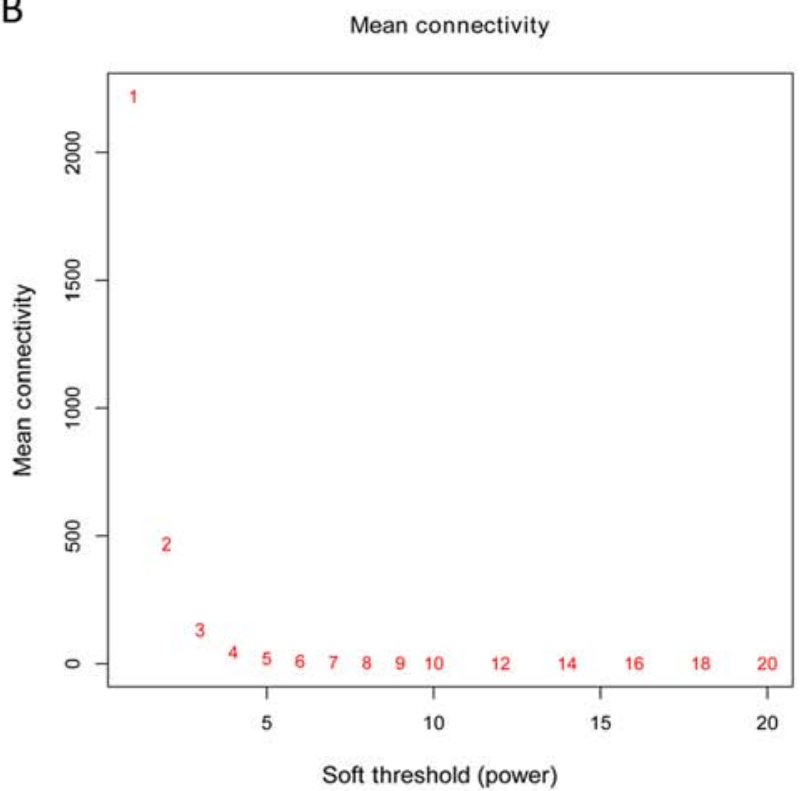

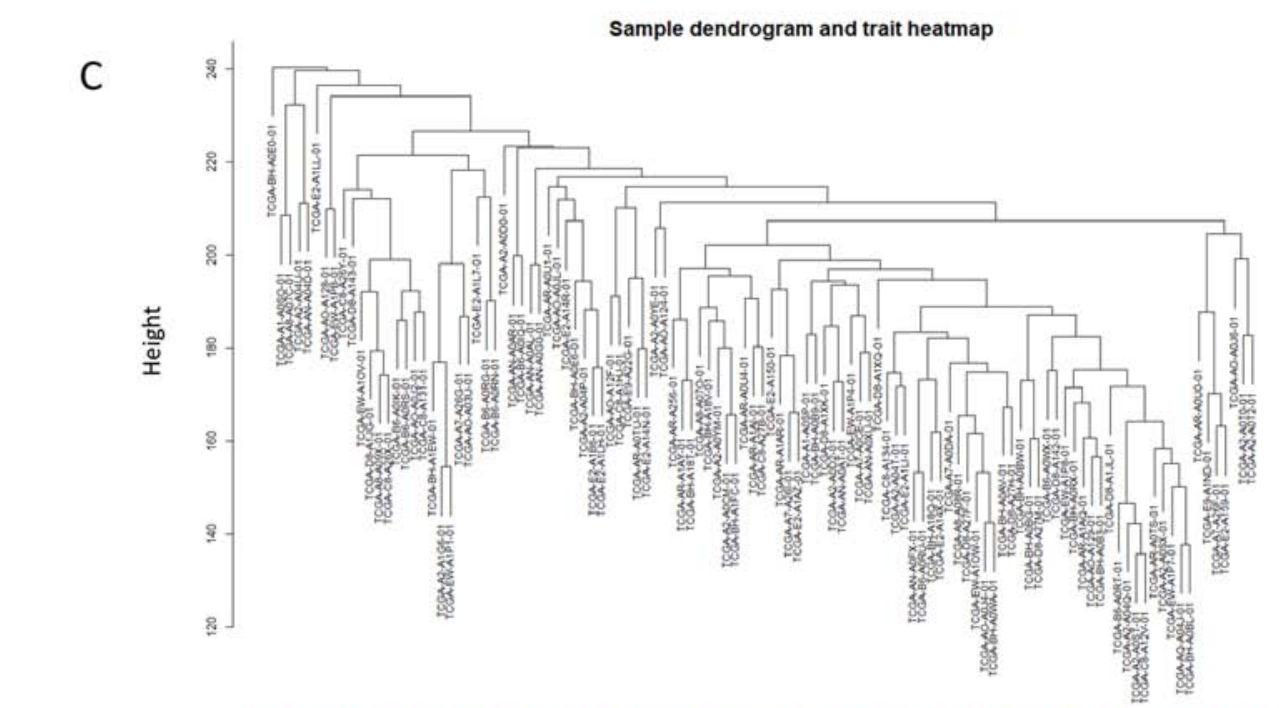

Age

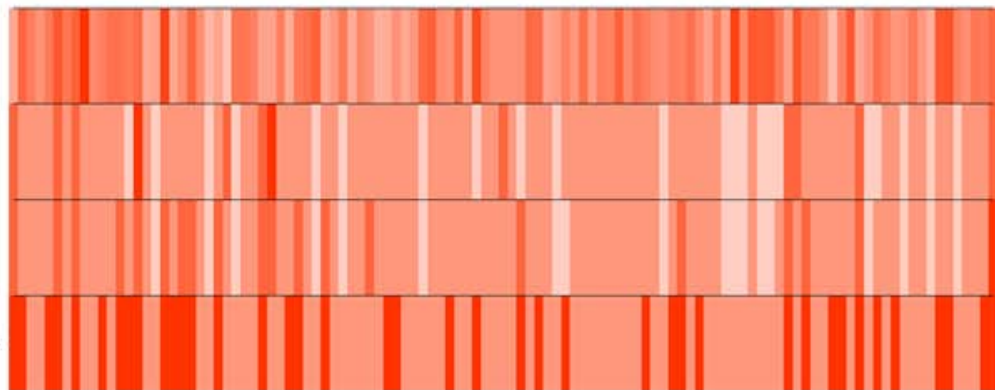

Figure 2. Soft-threshold power in WGCNA and K-means clustering of TNBC samples. (A) Relationship between scale-free topology model fit and soft-thresholds (powers). (B) Relationship between the mean connectivity and various soft-thresholds (powers). (C) Clustering dendrogram of TNBC tissues. WGNCA, weighted correlation network analysis; TNBC, triple-negative breast cancer.

Statistical analysis. Statistical analysis was performed using R (R Foundation for Statistical Computing; http://www.R-project. org/). The fold-change and Q-value (adjusted P-value) for para-tumor and tumor samples were calculated using the Limma package (9). A Q-value $<0.05$ was considered to be statistically significant. The overall survival analysis was conducted using the Survminer package (10), and the P-values in the KM curve were obtained using the log-rank test. The false discovery rate was set as 0.05 for analysis.

\section{Results}

WGCNA on RNAseq dataset of TNBC. In order to determine the co-expression network most highly associated with the 
A

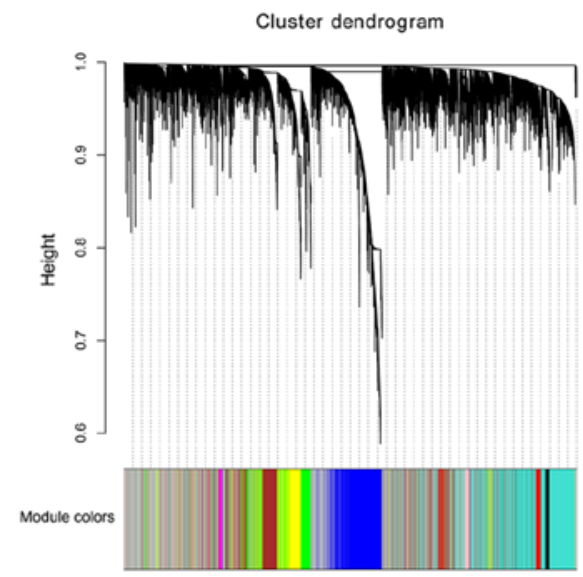

C

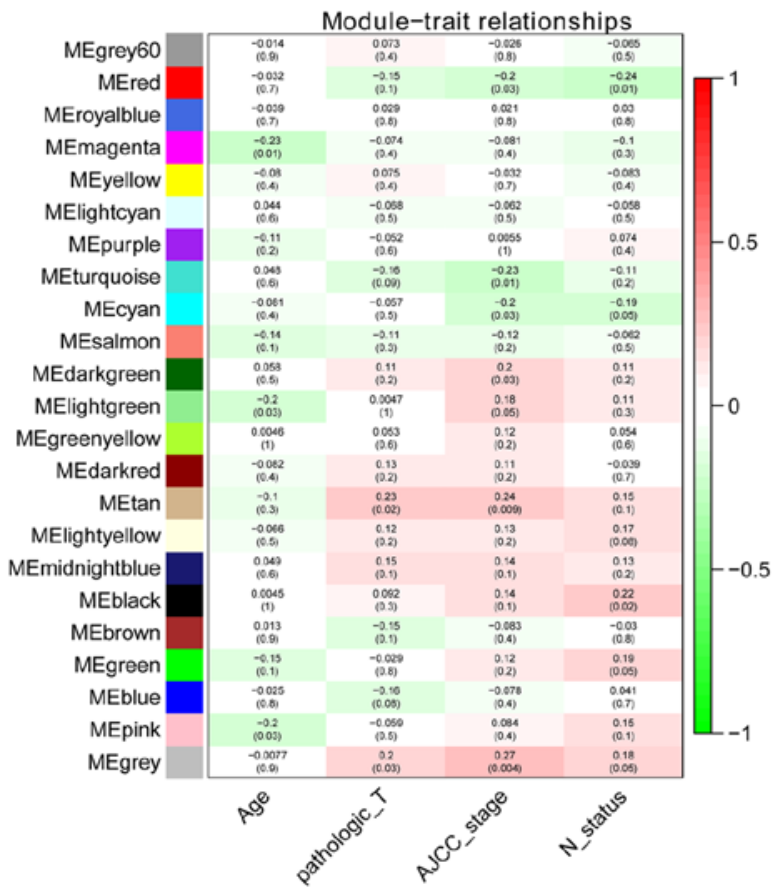

B

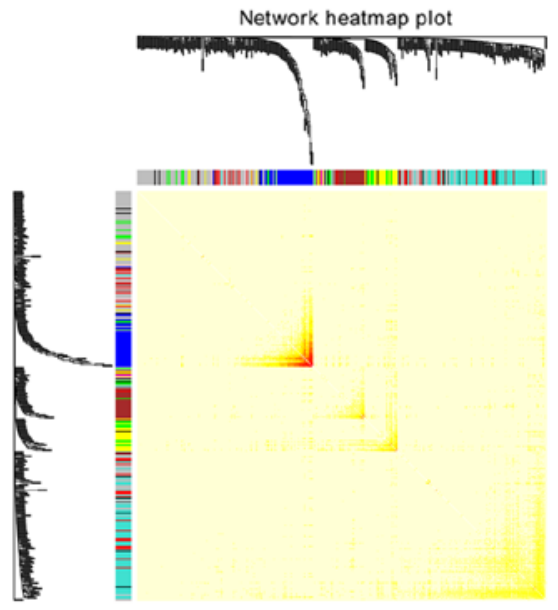

D

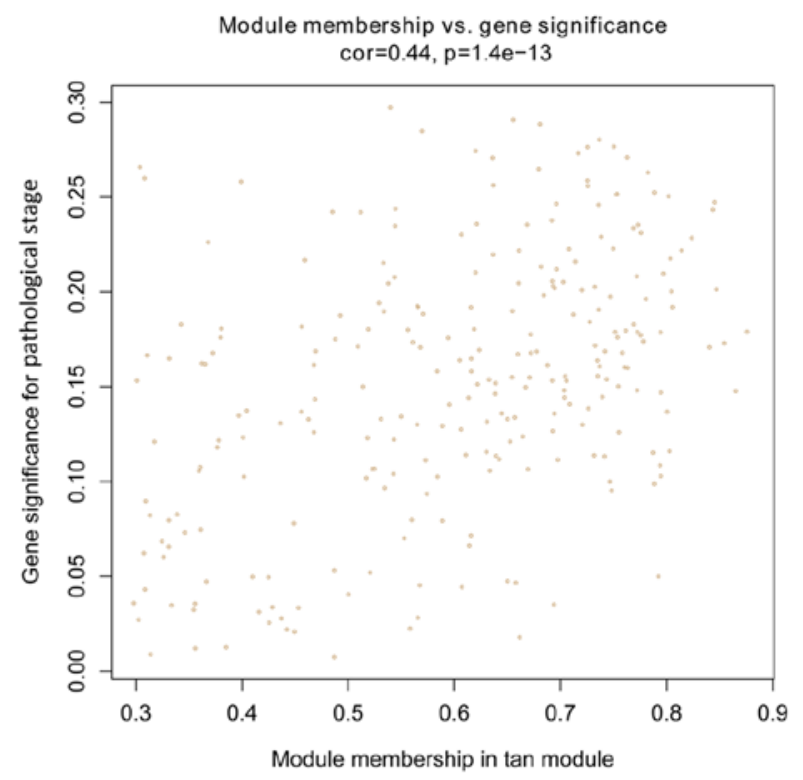

Figure 3. Identification of modules associated with the clinical traits of triple-negative breast cancer. (A) Dendrogram of modules identified by WGCNA. (B) Topological overlap matrix among detected genes from RNAseq. Genes with high intramodular connectivity are located at the tip of the module branches. (C) Heatmap of Module-clinical trait associations. (D) Scatterplot of gene significance for pathological stage vs. module membership in the tan module. WGNCA, weighted correlation network analysis.

progress and prognosis of TNBC, TNBCTCGA RNAseq datadownloaded from UCSC, was analyzed using WGCNA. The analysis showed TNBC clustering using the average linkage and Pearson's correlation methods. The scale-free network was constructed by raising the power of $\beta$ to 4 and by ensuring that the scale-free $\mathrm{R}^{2}$ reached 0.95 (Fig. $2 \mathrm{~A}$ and $\mathrm{B}$ ). The clustering dendrogram of TNBC tissues is shown in Fig. 2C.

A total of 23 modules were found to be clustered, and this gene clustering is displayed as a dendrogram in Fig. 3A. The weighted network of all genesis exhibited in a heat map, depicting the topological overlap matrix amongst the mRNA expression profiles (Fig. 3B). The tan module was determined using a trait-heat map to be the module with the strongest correlation with the pathological stage of TNBC (Fig. 3C). Fig. 3D illustrates the correlation of genes with pathological stage, as well as module membership (the correlation of genes with clusters) in the tan module. The results revealed that genes, which had high a correlation with tan modules were also strongly associated with the pathological stage of TNBC. Based on the cut-off criteria ( $G S \mid>0.4), 129$ genes with high connectivity were selected for the construction of the co-expression network. The inner connectivity in the tan module with the threshold ( $|G S|>0.4$ ) was plotted. This showed strong co-expression relationships in the tan module (Fig. 4).

GO and Kyoto Encyclopedia of Genes and Genomes (KEGG) Analysis. The genes in the tan module were divided into three groups (biological process, cellular component, and molecular function), which were then analyzed using GO and KEGG analysis. In the biological process group, the most enriched genes concerned epigenetic regulation, cell metabolism, DNA repair and mRNA processing (Fig. 5A). The genes in the cellular component group that were most enriched 


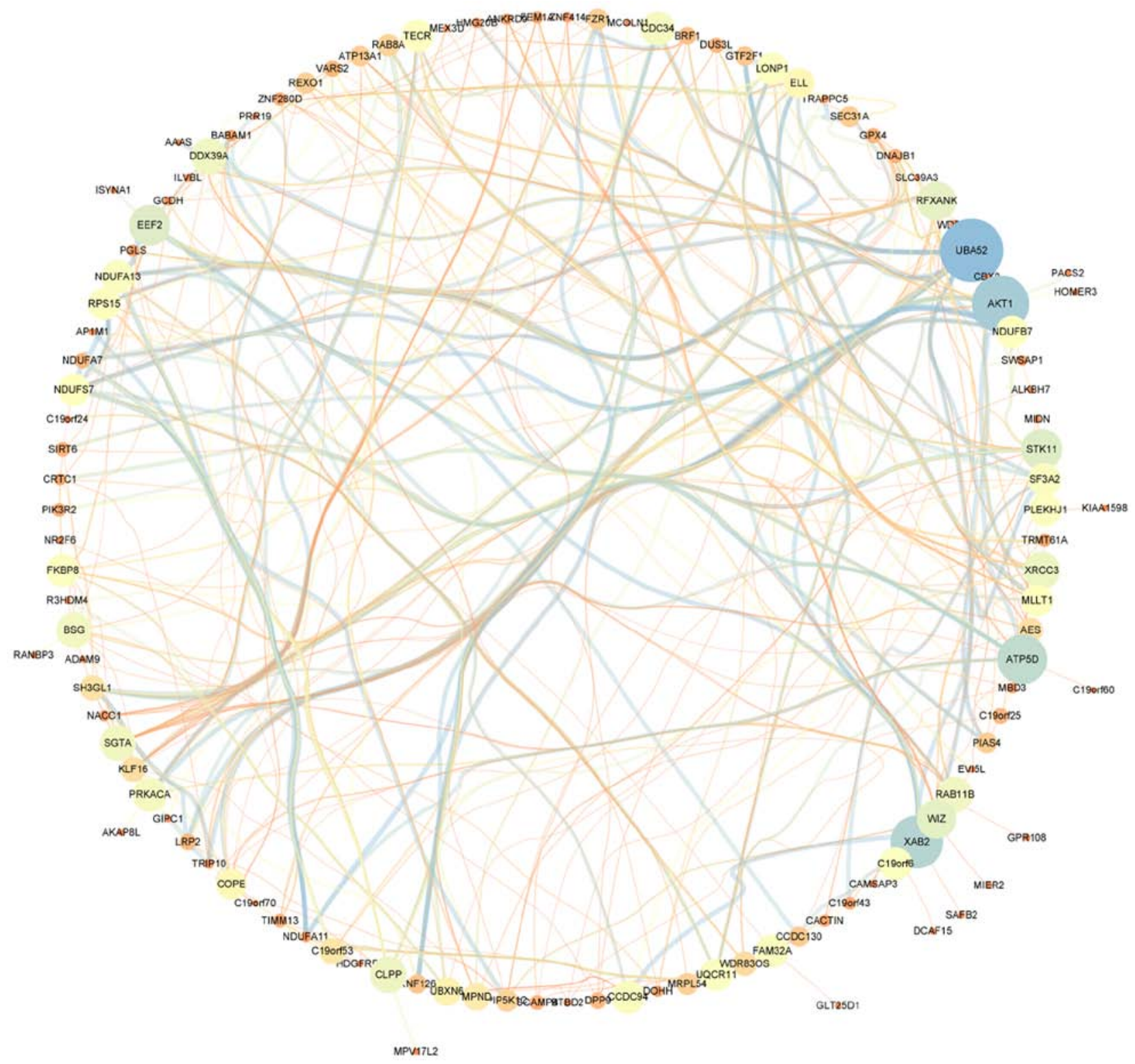

Figure 4. Innerconnectivity in the tan module. Node size indicates the betweenness centrality, which reflects the amount of control that this node exerts over the interactions of other nodes in the network. Edge sizes depict the weight between connected genes.

comprised 'mitochondrial protein complex', 'endosomal part' and 'phagocytic cup' (Fig. 5B). The most enriched genes in the molecular function group were in 'cadherin binding', 'transcription corepressor activity' and 'SH3 domain binding', (Fig. 5C). KEGG pathway analysis indicated that 'autophagy' and 'AMPK signaling pathway' were involved in the regulation of genes in the tan module (Fig. 5D).

DEG analysis of TNBC tissues. The DEGs were obtained from the analysis of RNAseq datasets, and a total of 2,169 significant genes were identified ( $\mathrm{Q}$-value $<0.05$; fold change $>2$ ) from the comparison of TNBT tissues and para-tumor tissues. The volcano plot indicates the fold-change and P-value of DEGs (Fig. 6A). The overlap between the genes discovered in DEG analysis and the genes in the tan module comprised 42 genes, which were selected as hub-genes. Among the 42 genes, 5 of which showed high correlation between expression level and survival probability, and were used for further analysis.
Survival and expression of the 5 selected genes. From the aforementioned hub-genes, 5 genes [GIPC PDZ domain containing family member 1 (GIPC1), hes family bHLH transcription factor 6 (HES6), calmodulin-regulated spectrin-associated protein family member 3 (KIAA1543), myosin light chain kinase 2 (MYLK2) and peter pan homolog (PPAN)] were selected to explore their association with the pathological progress. The unsupervised clustering results illustrate the co-expression of the 5 genes in the tan module and the extent of correlation between the 5 genes in TNBC was determined. Fig. 6B indicates high correlation between HES6 and GIPC1, and also between PPAN and GIPC1. The expression level of the genes in different pathological stages varied, and tended to be higher in more progressed pathological stages (Fig. 7). The expression levels of the 5 genes in tumor and para-tumor tissues were also plotted in Fig. 8, and showed significantly higher expression in tumor tissues $(\mathrm{P}<0.01)$. The ROC curves indicate that GIPC1, HES6, KIAA1543, MYLK2 and PPAN 
A
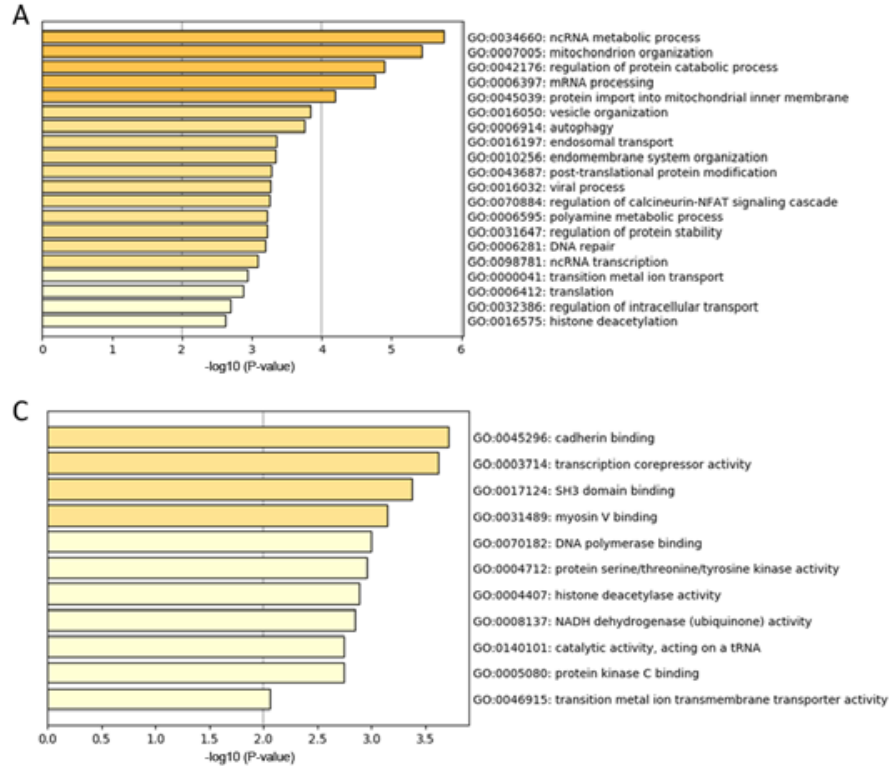

B
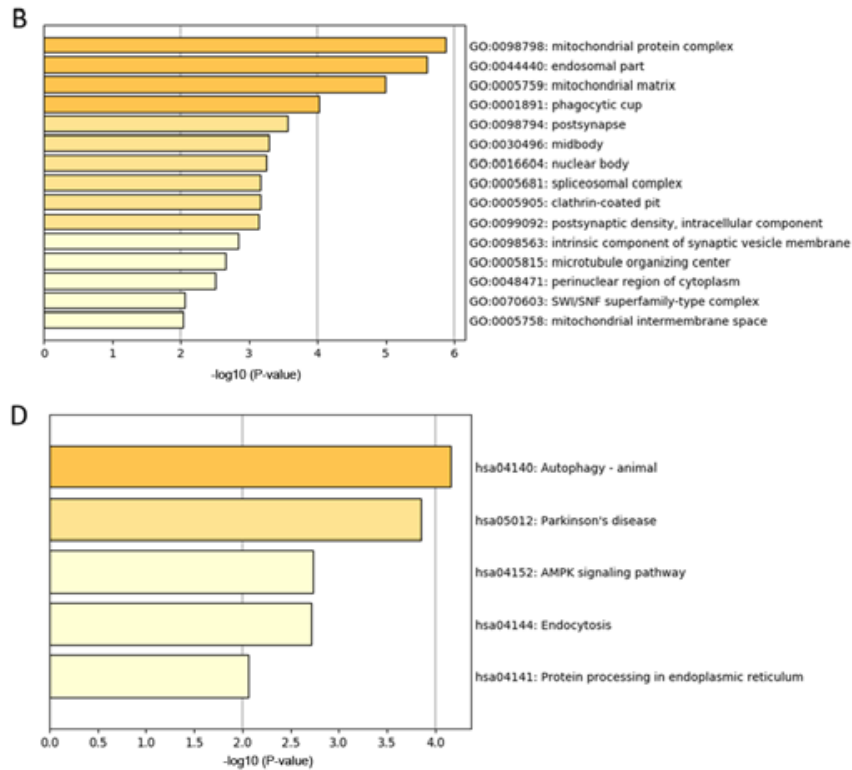

Figure 5. Gene ontology and pathway enrichment analysis of genes in the tan module. (A) Biological process analysis. (B) Cellular component analysis. (C) Molecular function analysis. (D) KEGG pathway analysis. KEGG, Kyoto Encyclopedia of Genes and Genomes.

A

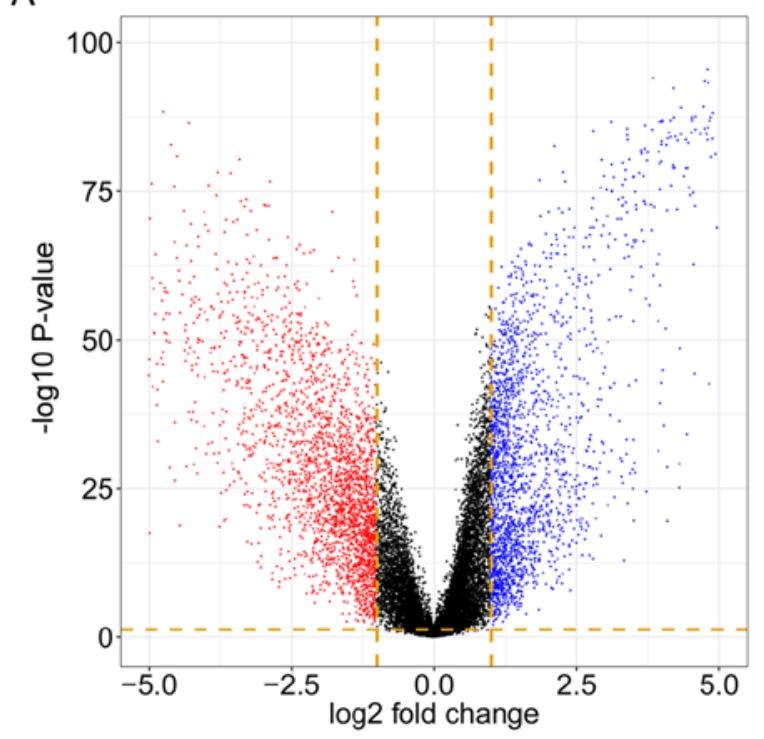

B

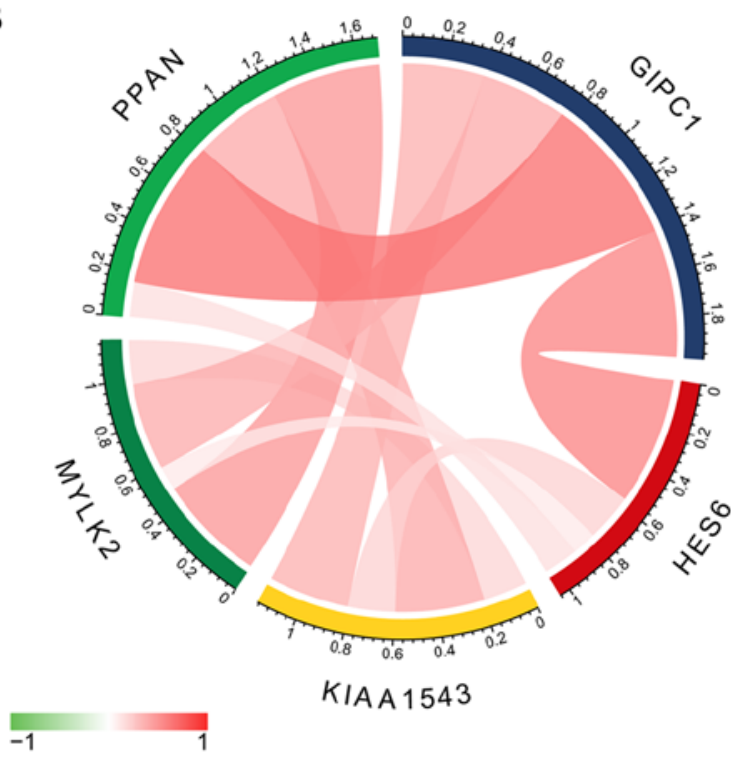

Figure 6. Differentially expressed gene analysis and correlation of the 5 selected genes. (A) The volcano plot for differentially expressed genes in the tumor group and the para-tumor group. (B) Correlation between the 5 selected genes.

all exhibited high diagnostic accuracy for the identification of para-tumor and tumor tissues (Fig. 9). The KM curves illustrate that the expression levels of the 5 genes were associated with the OS of patients with TNBC (Fig. 10). Furthermore, high-expression groups of KIAA1543, MYLK2 and PPAN were significantly associated with lower OS times when compared with low-expression of the same proteins $(\mathrm{P}<0.05)$.

\section{Discussion}

Breast cancer is an umbrella term summarizing carcinomas originating from the breast, and is one of the most prevalent cancer types worldwide. TNBCs are described as breast cancers that simultaneously lack expression of the ER, PR and HER 2 genes. Triple-negative tumors represent $80 \%$ of basal-like molecular breast cancers (1). They have less favorable outcomes compared with other molecular subtypes of breast cancer, as they are commonly associated with a higher risk of early relapse and poor survival (11). Currently, no drug is available that specifically targets TNBC, and the results of chemotherapy are unsatisfactory. The molecular characteristics of TNBC include disruption of BRCA1 DNA repair associated (BRCA)-1 function $(12,13)$. The low expression level of BRCA-1 and inhibitor of DNA binding 4, HLH protein (ID4) lead to the change from homologous repair (HR) to non-homologous end joining (NHEJ) or single-strand 
A

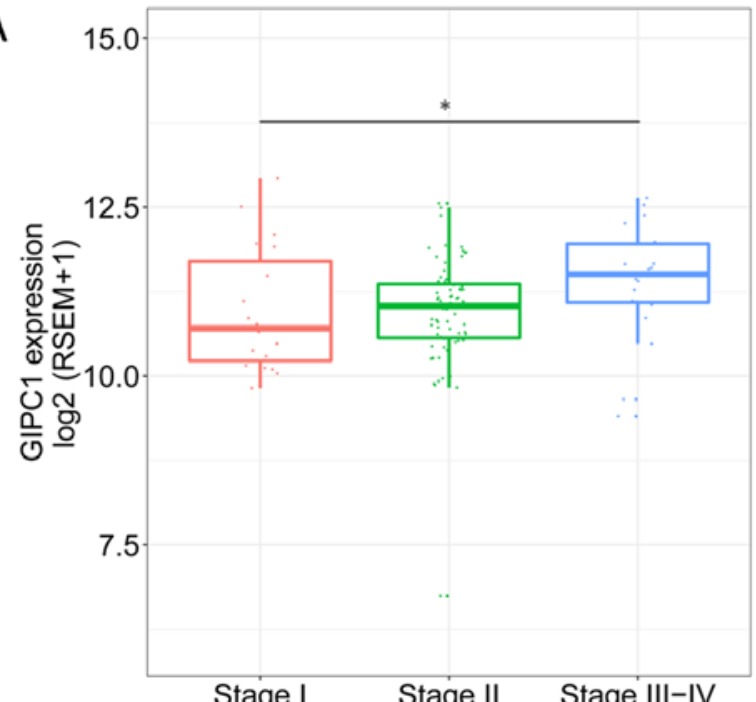

C

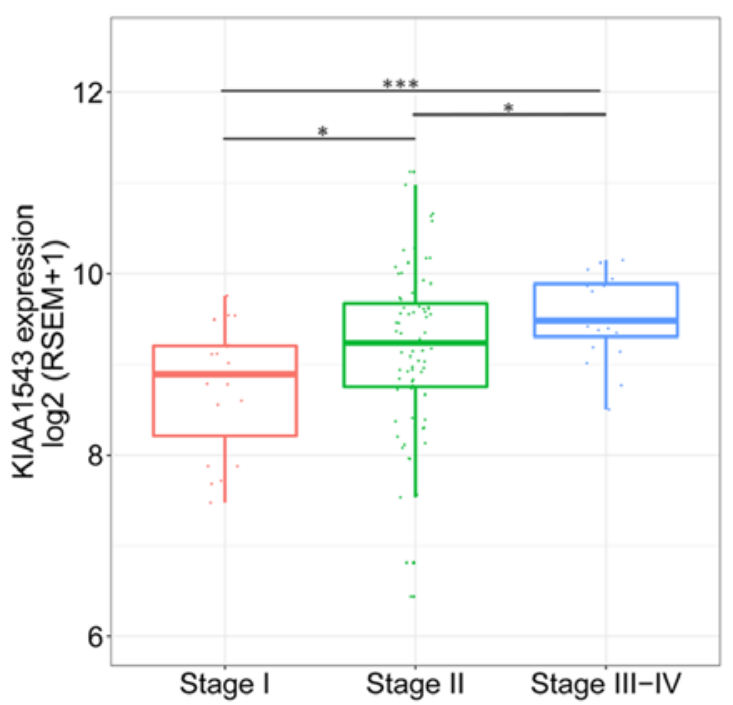

E

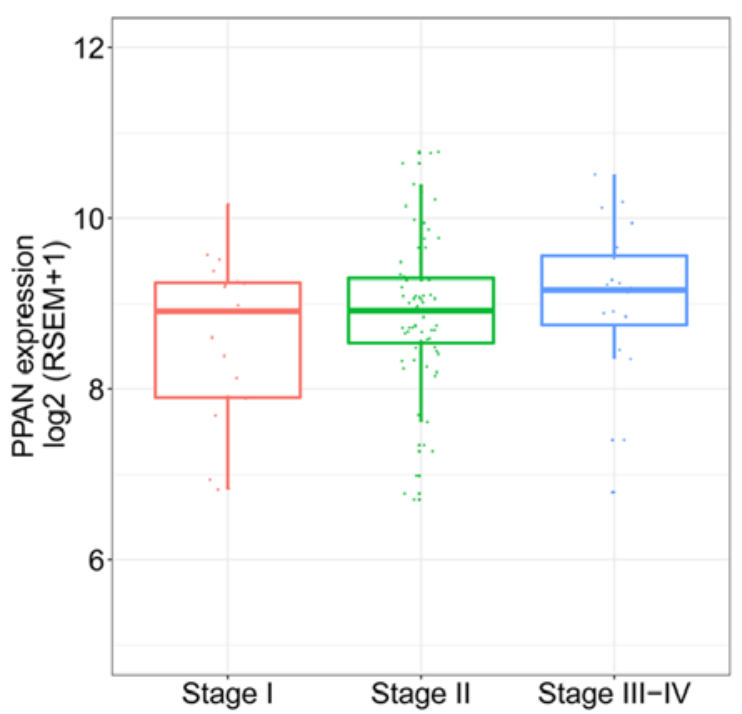

B

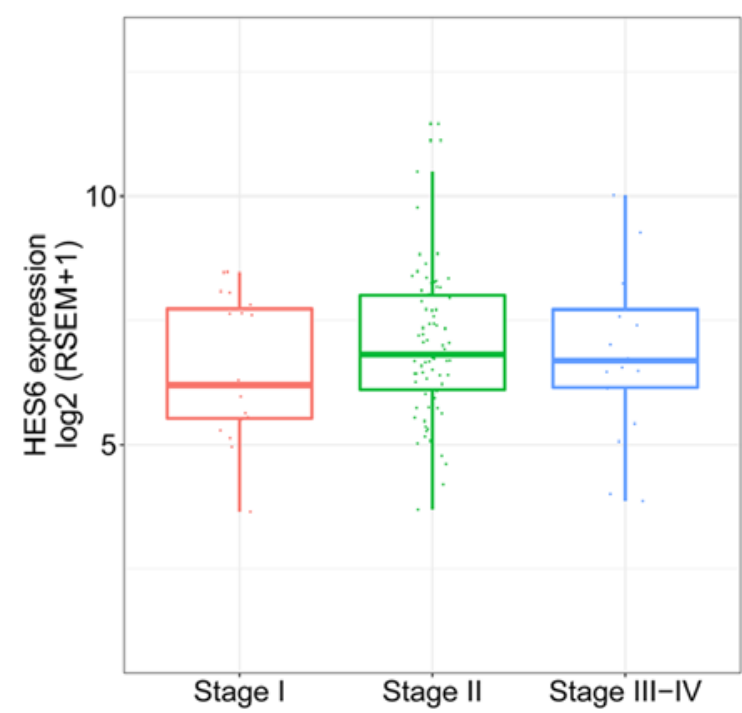

D

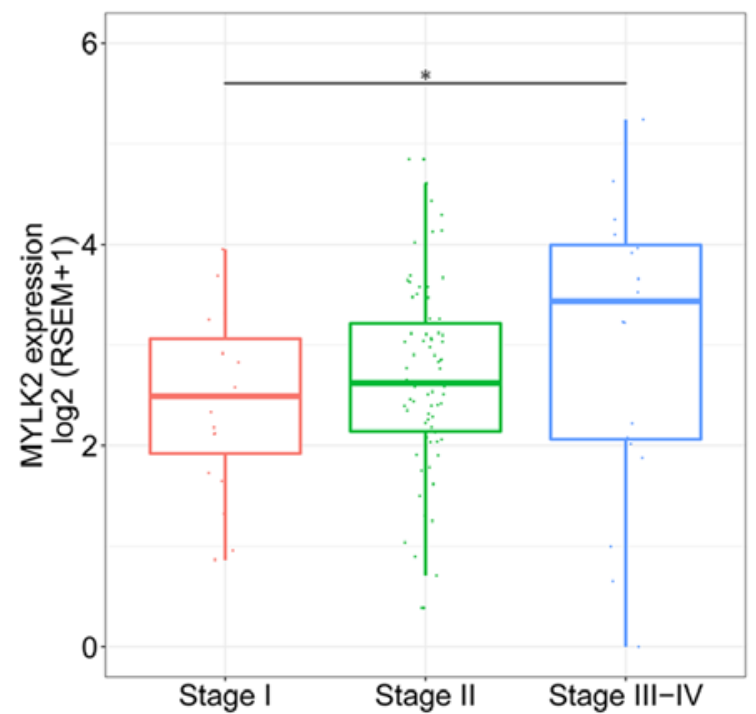

Figure 7. Expression of GIPC1, HES6, KIAA1543, MYLK2, and PPAN in different pathological stages of triple-negative breast cancer. (A) GIPC1. (B) HES6. (C) KIAA1543. (D) MYLK2. (E) PPAN. "P<0.05 and ${ }^{* * *} \mathrm{P}<0.005$ indicated a significant difference between groups. Wilcoxon signed-rank test was used to evaluate the statistical significance of differences. GIPC1, GIPC PDZ domain containing family member 1; HES6, hes family bHLH transcription factor 6; KIAA1543, calmodulin-regulated spectrin-associated protein family member 3; MYLK2, myosin light chain kinase 2; PPAN, peter pan homolog. 
A

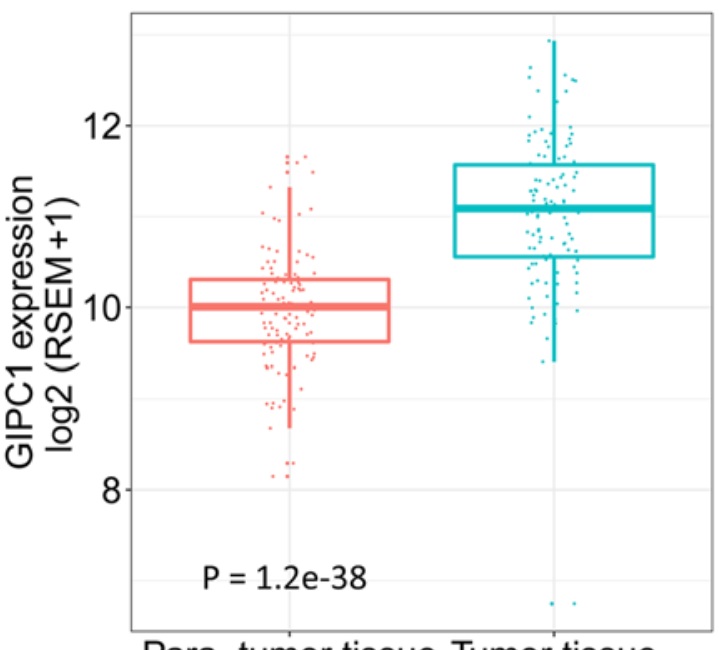

Para-tumor tissue Tumor tissue

C

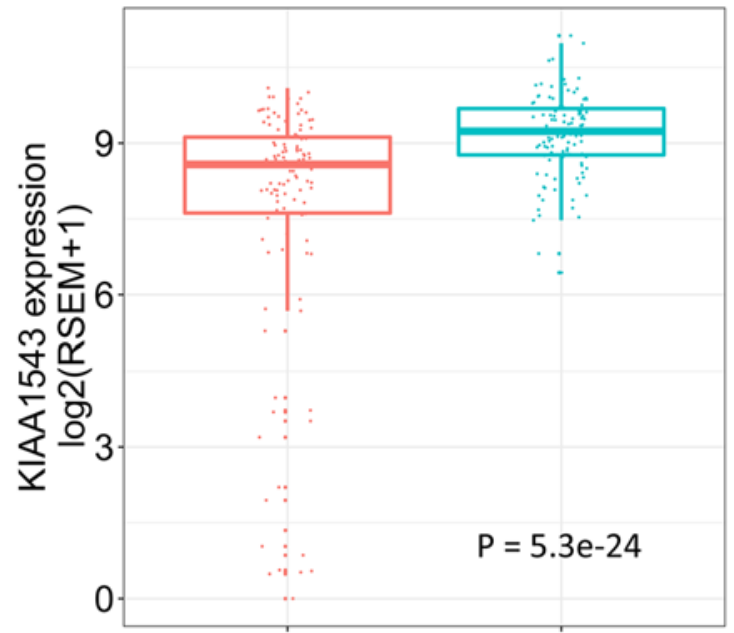

Para-tumor tissue Tumor tissue

$E$

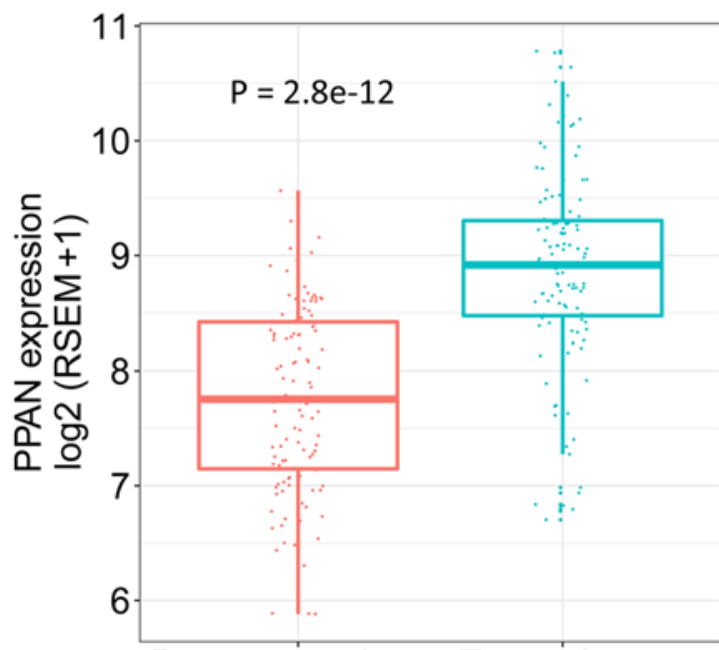

B

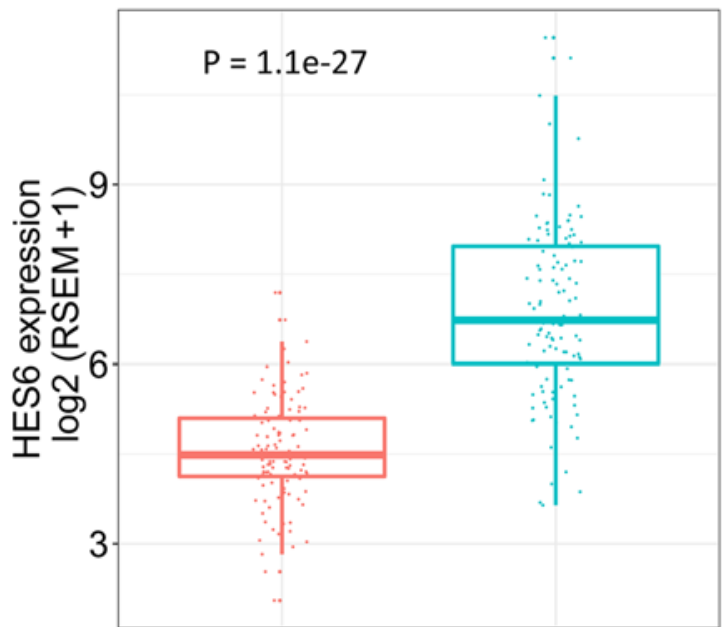

Para-tumor tissue Tumor tissue

D

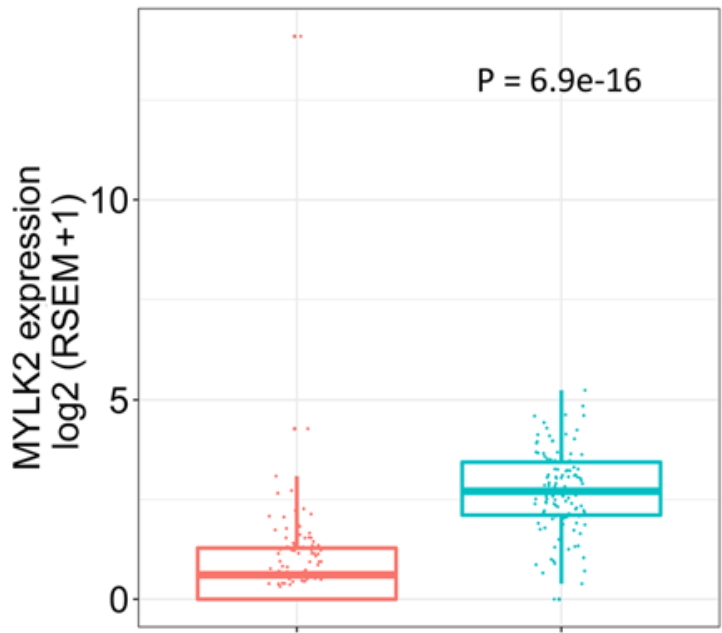

Para-tumor tissue Tumor tissue

Para-tumor tissue Tumor tissue

Figure 8. Expression of GIPC1, HES6, KIAA1543, MYLK2 and PPAN in para-tumor and tumor tissues. (A) GIPC1. (B) HES6. (C) KIAA1543. (D) MYLK2. (E) PPAN. Adjusted Q value, determined using the R Limma package, was used to evaluate the statistical significance of differences. GIPC1, GIPC PDZ domain containing family member 1; HES6, hes family bHLH transcription factor 6; KIAA1543, calmodulin-regulated spectrin-associated protein family member 3; MYLK2, myosin light chain kinase 2; PPAN, peter pan homolog.

annealing (SSA) pathways $(14,15)$. HR is the most important DNA repair mechanism in healthy tissues and maintains genomic stability. However, the change to NHEJ (the alternative, more error-prone DNA-repair mechanism) can lead to 
A

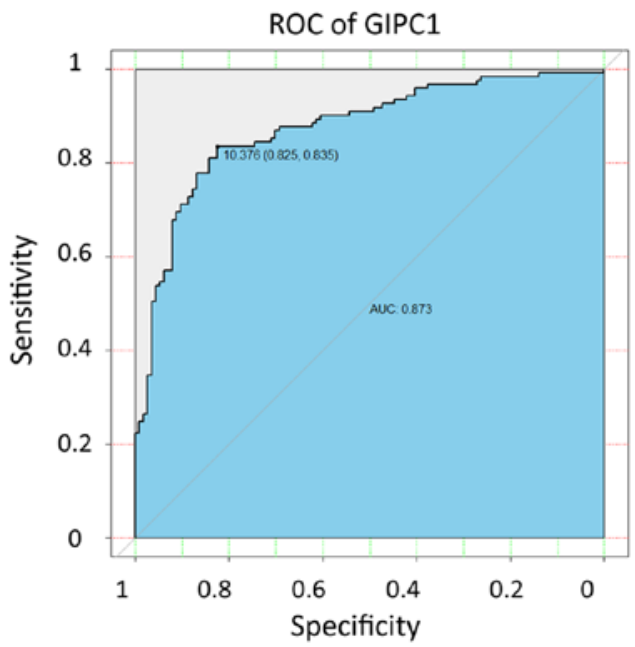

$\mathrm{C}$

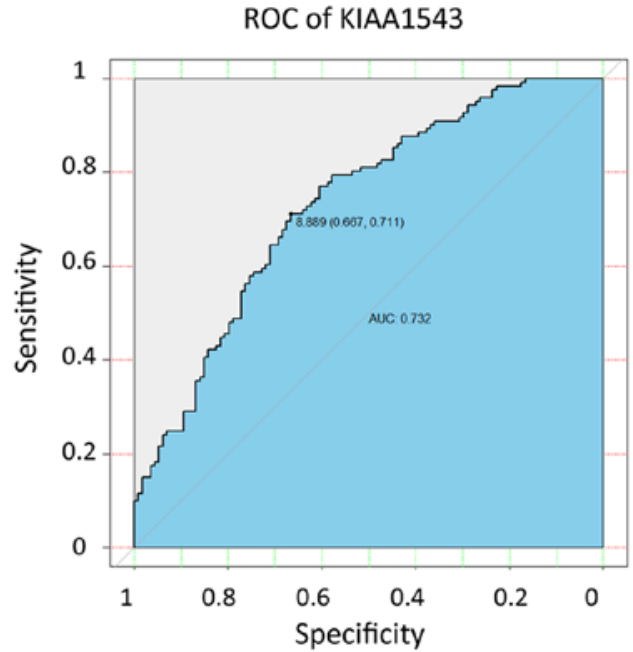

$E$

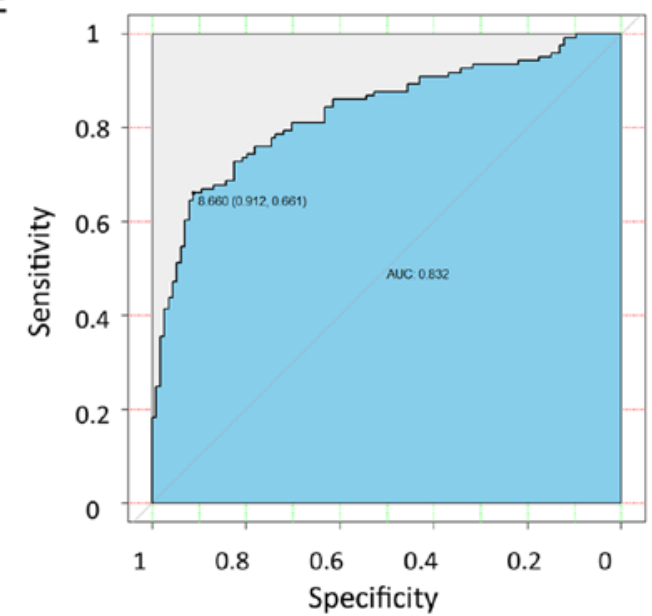

B

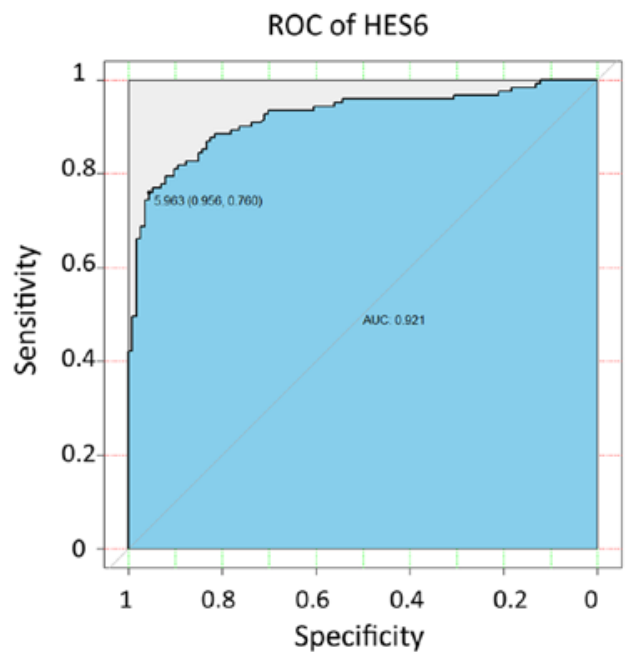

D

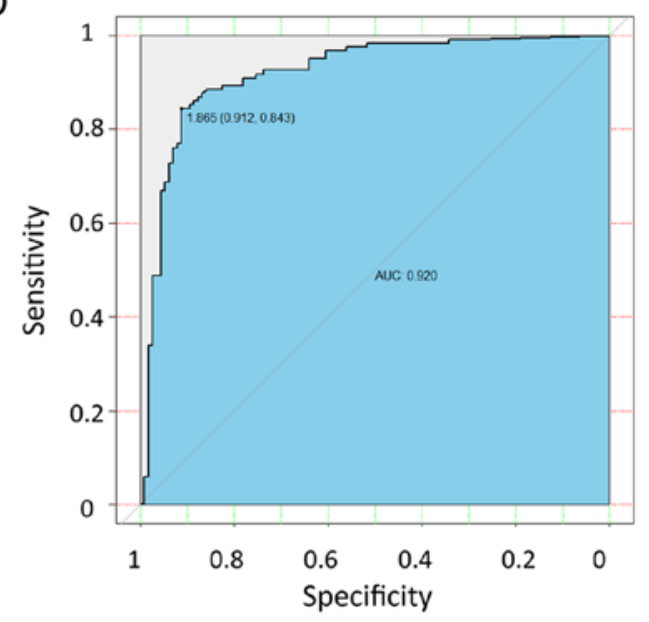

Figure 9. ROC curve of GIPC1, HES6, KIAA1543, MYLK2 and PPAN expression levels to differentiate tumor and para-tumor tissues. (A) GIPC1. (B) HES6. (C) KIAA1543. (D) MYLK2. (E) PPAN. ROC, receiver operating characteristic; GIPC1, GIPC PDZ domain containing family member 1; HES6, hes family bHLH transcription factor 6; KIAA1543, calmodulin-regulated spectrin-associated protein family member 3; MYLK2, myosin light chain kinase 2; PPAN, peter pan homolog.

genetic instability in tumor tissues. The inhibitor olaparib, which targets PARP-1, can block the NHEJ of breast cancer types and ultimately inhibit DNA repair (16). Moreover, epigenetic dysregulation is well established as having a crucial role in cancer pathology and progression $(17,18)$, and the current study identified enrichment of epigenetic regulation in TNBC.

However, the molecular characteristics of TNBC are not well understood. Exploring the mechanisms involved in the progress and prognosis of TNBC would be helpful for improving diag- 
A
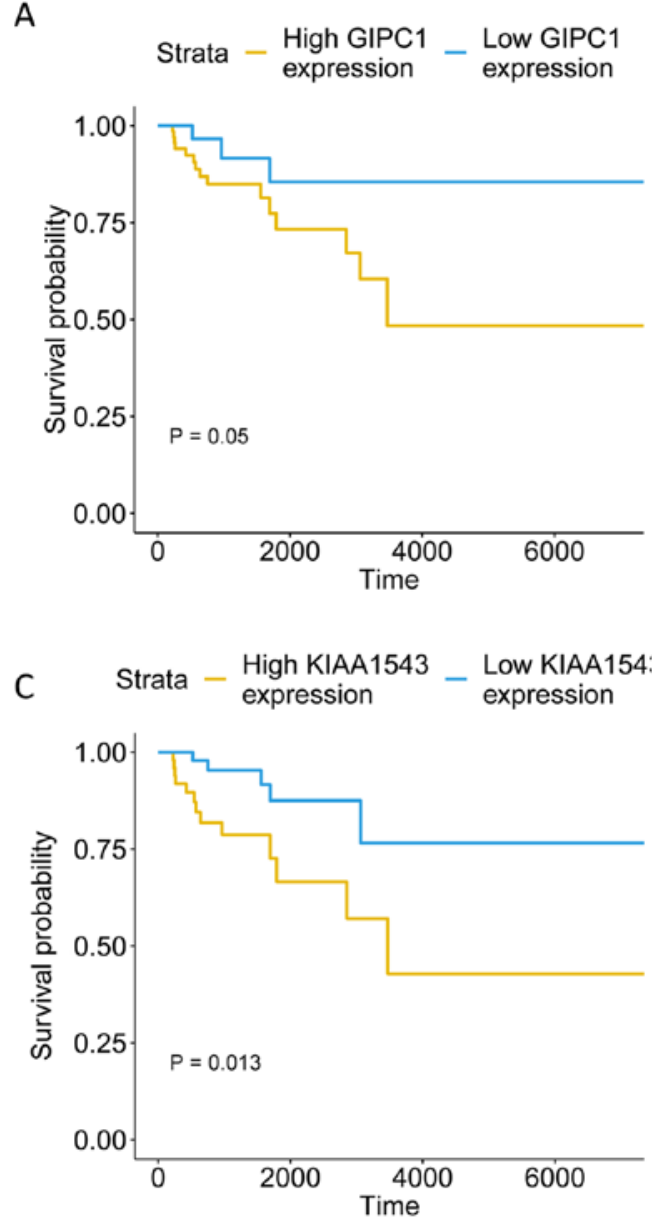

$\mathrm{E}$

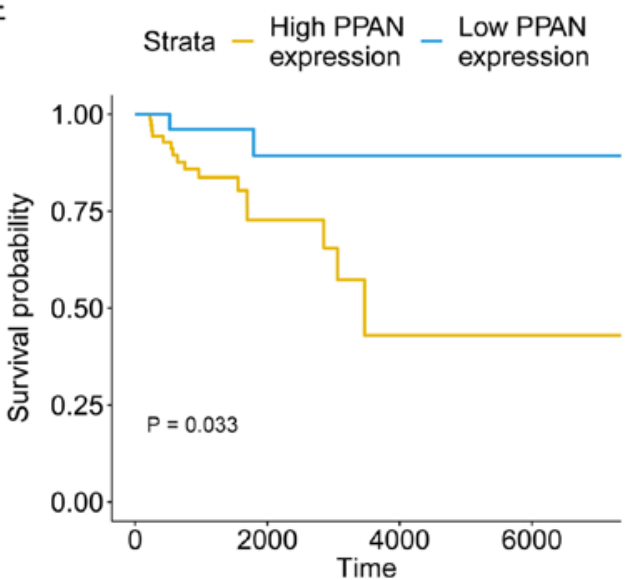

B
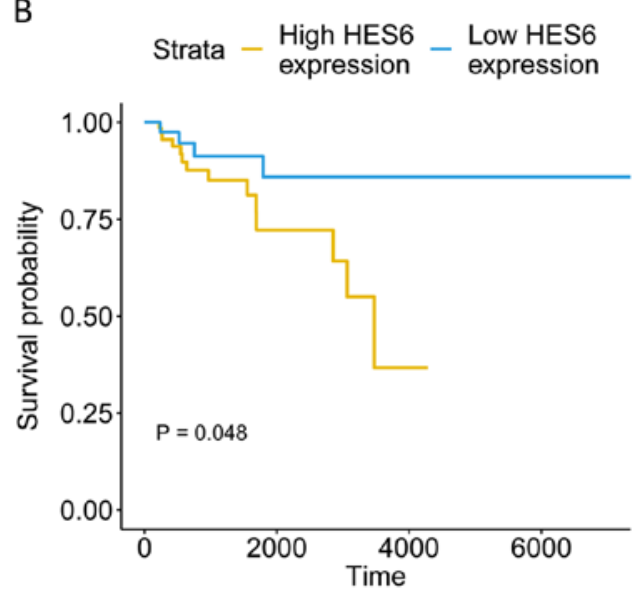

D Strata $-\underset{\text { expression }}{\text { High MYLK2 }}-\underset{\text { Low MYLK2 }}{\text { expsion }}$

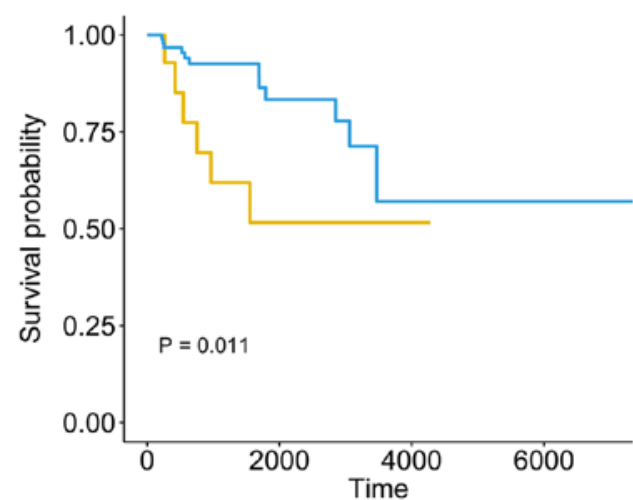

Figure 10. Overall survival of the 5 hub-genes in triple-negative breast cancer. (A) GIPC1. (B) HES6. (C) KIAA1543. (D) MYLK2. (E) PPAN. GIPC1, GIPC PDZ domain containing family member 1; HES6, hes family bHLH transcription factor 6; KIAA1543, calmodulin-regulated spectrin-associated protein family member 3; MYLK2, myosin light chain kinase 2; PPAN, peter pan homolog.

nosis and treatment, and new targets or biomarkers are required. The present study determined a number of candidates using TGCA RNAseq datasets downloaded from UCSC database to identify promising biomarkers related to TNBC progression. WGCNA is a commonly used bioinformatics analysis tool used to identify the key modules and genes, which are associated with specific clinical traits. A study applied WGCNA analysis in breast cancer, identifying the association between cyclin B2 (CCNB2), F-box protein 5 (FBXO5), kinesin family member 4A (KIF4A), minichromosomal maintenance 10 replication initiation factor (MCM10) and TPX2 microtubule nucleation factor (TPX2) expression, and the survival of breast cancer patients (19). Another study used WGCNA to reveal the association between ATRX chromatin remodeler (ATRX) and TNBC (20). In the present study, WGCNA was performed to determine the association between American Joint Committee on Cancer-TNM stage and the gene co-expression module. By overlapping the genes from the tan module and DEG analysis, 
42 hub genes were identified. Of these 42 genes, GIPC1, HES6, KIAA1543, MYLK2 and PPAN were selected to validate the strength of association with TNBC progression.

GIPC1 (also known as C19orf3) is a cytoplasmic protein that acts as an adaptor protein, linking receptor interactions to intracellular signaling pathways, including cell cycle regulation (21). GIPC1 protein is highly expressed both in cultured human breast cancer cells and in primary and metastatic tumor tissues (22). It is a cancer-associated immunogenic antigen in breast cancer which is also associated with bone metastasis development in breast cancer (23). The functions of GIPC1 include the regulation of apoptotic cell death, $\mathrm{G}_{2}$ cell-cycle arrest, modified cell adhesion and the migration of breast cancer cells (24). In the present analysis, GIPC1 was significantly upregulated in both triple-negative breast tumor tissue and a progressed pathological stage.

HES6 encodes a subfamily of basic helix-loop-helix transcription repressors with homology to the Drosophila enhancer of split genes (25). The overexpression of HES6 is found in metastatic carcinomas of different origins. Hes6 ectopic expression stimulates cell proliferation, not only in breast cancer T47D cells, but also in breast tumor growth in xenografts. The overexpression of HES6 also led to the induction of E2F transcription factor 1, a crucial target gene for the transcriptional repressor HES1 (26). Furthermore, the ER $\alpha$-negative breast cancer cell lines MDA-MB-231 and SKBR3express 4 to 10 times higher levels of Hes-6 mRNA, compared with ER $\alpha$-positive T47D and MCF-7 cells. The aforementioned studies complement the present findings of an association between HES6 expression and estrogen in TNBC.

KIAA1543, also known as Nezha, was shown to bridge the minus ends of non-centrosome anchored microtubules with p120, which plays a crucial role in stabilizing cadherin-catenin mediated cell-cell adhesion complexes $(27,28)$. KIAA1543 is also involved in epithelial-mesenchymal transition, and mediates the interaction between cadherin and microtubules. It is overexpressed in invasive lobular carcinomas but its role is poorly characterized $(29,30)$. The underlying mechanisms of KIAA1543 on TNBCs need to be further established. The current study found KIAA1543 to be significantly upregulated in TNBC tissues and at advanced pathological stages. Moreover, high KIAA1543 expression was associated with poor OS in TNBC patients.

MYLK2 encodes a calcium/calmodulin-dependent serine/threonine kinase (31). A somatic mutation in, or amplification of, MYLK2 has been detected in several cancer tissues, compared with normal tissues (31). Moreover, proteomic analyses conducted on serum protein profiling results revealed the upregulation of MYLK2 in pancreatic cancer patients (32). The present study showed that in TNBC tissues, MYLK2 was overexpressed and was also associated with the poor patient OS.

PPAN encodes an evolutionarily conserved protein similar to the Drosophila gene peter pan. A signature inferred from Drosophila mitotic genes revealed an association between PPAN expression and the survival of breast cancer patients (33). Tumor patients with high expression levels of PPAN have been shown to respond more favorably to treatment with anti-EGFR antibodies such as cetuximab (34). The data presented reveal a significant association between high PPAN expression and a poor OS in patients with TNBC.
Taken together, WGCNA and DEG analysis on RNAseq datasets from TNBC tissues revealed that the tan module was the most significantly associated with AJCC-TNM stage. The genes in the tan module showed high inter-connectivity with the co-expression network. Furthermore, GO and KEGG analysis revealed the enrichment of the genes in the related terms from GO. The overlap between the module and DEG analysis identified 42 genes, 5 of which were negatively associated with the OS of patients with TNBC. Therefore, the current study provides 5 novel biomarkers for TNBC, which exhibit potential as targets in the diagnosis and treatment of the disease.

\section{Acknowledgements}

Not applicable.

\section{Funding}

This study was supported by the Jinhua technology division fund (grant no. 2018-4-120).

\section{Availability of data and materials}

The datasets generated and/or analyzed during the present study are available in the University of California, Santa Cruz repository, (https://xenabrowser.net/datapages/).

\section{Authors' contributions}

$\mathrm{MB}, \mathrm{JW}, \mathrm{LB}$ and $\mathrm{KZ}$ conceived the experiment design. LB, $\mathrm{JW}, \mathrm{KZ}$ and TG performed the data analysis. JW and $\mathrm{KZ}$ revised the manuscript. $\mathrm{LB}, \mathrm{TG}, \mathrm{JW}, \mathrm{KZ}$ and $\mathrm{MB}$ wrote the manuscript.

\section{Ethics approval and consent to participate}

Not applicable.

\section{Patient consent for publication}

Not applicable.

\section{Competing interests}

The authors declare that they have no competing interests.

\section{References}

1. Foulkes WD, Smith IE and Reis-Filho JS: Triple-negative breast cancer. N Engl J Med 363: 1938-1948, 2010.

2. Dawood S: Triple-negative breast cancer: Epidemiology and management options. Drugs 70: 2247-2258, 2010.

3. Dent R, Trudeau M, Pritchard KI, Hanna WM, Kahn HK, Sawka CA, Lickley LA, Rawlinson E, Sun P and Narod SA: Triple-negative breast cancer: Clinical features and patterns of recurrence. Clin Cancer Res 13: 4429-4434, 2007.

4. Liedtke C, Mazouni C, Hess KR, André F, Tordai A Mejia JA, Symmans WF, Gonzalez-Angulo AM, Hennessy B, Green M, et al: Response to neoadjuvant therapy and long-term survival in patients with triple-negative breast cancer. J Clin Oncol 26: 1275-1281, 2008.

5. Langfelder $P$ and Horvath S: WGCNA: An R package for weighted correlation network analysis. BMC Bioinformatics 9: $559,2008$. 
6. Wang Y, Zhang Q, Gao Z, Xin S, Zhao Y, Zhang K, Shi R and Bao X: A novel 4-gene signature for overall survival prediction in lung adenocarcinoma patients with lymph node metastasis. Cancer Cell Int 19: 100, 2019.

7. Huang K, Maruyama T and Fan G: The naive state of human pluripotent stem cells: A synthesis of stem cell and preimplantation embryo transcriptome analyses. Cell Stem Cell 15: 410-415, 2014.

8. Wang Y, Xin S, Zhang K, Shi R and Bao X: Low GAS5 levels as a predictor of poor survival in patients with lower-grade gliomas. J Oncol 2019: 1785042, 2019.

9. Smyth GK. Limma: linear models for microarray data Bioinformatics and computational biology solutions using $\mathrm{R}$ and Bioconductor. Statistics for Biology and Health. Gentleman R., Carey V.J., Huber W., Irizarry R.A., Dudoit S. (Eds). Springer, New York, NY, pp. 397-420, 2005.

10. Kassambara A, Kosinski M and Biecek P: Survminer: Drawing Survival Curves using'ggplot2'. R package version 03. 2017;1.

11. Haffty BG, Yang Q, Reiss M, Kearney T, Higgins SA, Weidhaas J, Harris L, Hait W and Toppmeyer D: Locoregional relapse and distant metastasis in conservatively managed triple negative early-stage breast cancer. J Clin Oncol 24: 5652-5657, 2006.

12. Atchley DP, Albarracin CT, Lopez A, Valero V, Amos CI, Gonzalez-Angulo AM, Hortobagyi GN and Arun BK: Clinical and pathologic characteristics of patients with BRCA-positive and BRCA-negative breast cancer. J Clin Oncol 26: 4282-4288, 2008

13. Cleator S, Heller W and Coombes RC: Triple-negative breast cancer: Therapeutic options. Lancet Oncol 8: 235-244, 2007.

14. Powell SN and Kachnic LA: Roles of BRCA1 and BRCA2 in homologous recombination, DNA replication fidelity and the cellular response to ionizing radiation. Oncogene 22: 5784-5791, 2003

15. Evers B, Helleday $\mathrm{T}$ and Jonkers J: Targeting homologous recombination repair defects in cancer. Trends Pharmacol Sci 31: 372-380, 2010

16. Wang $\mathrm{M}, \mathrm{Wu} \mathrm{W}, \mathrm{Wu} \mathrm{W}$, Rosidi $\mathrm{B}$, Zhang $\mathrm{L}$, Wang $\mathrm{H}$ and Iliakis G: PARP-1 and Ku compete for repair of DNA double strand breaks by distinct NHEJ pathways. Nucleic Acids Res 34: 6170-6182, 2006.

17. Wang Y, Deng H, Xin S, Zhang K, Shi R and Bao X: Prognostic and predictive value of three DNA methylation signatures in lung adenocarcinoma. Front Genet 10: 349, 2019.

18. Baylin SB and Jones PA: Epigenetic determinants of cancer. Cold Spring Harb Perspect Biol 8: a019505, 2016.

19. Tang J, Kong D, Cui Q, Wang K, Zhang D, Gong Y and Wu G: Prognostic genes of breast cancer identified by gene co-expression network analysis. Front Oncol 8: 374, 2018.

20. Lan L, Xu B, Chen Q, Jiang J and Shen Y: Weighted correlation network analysis of triple-negative breast cancer progression: Identifying specific modules and hub genes based on the GEO and TCGA database. Oncol Lett 18: 1207-1217, 2019.

21. Valdembri D, Caswell PT, Anderson KI, Schwarz JP, König I, Astanina E, Caccavari F, Norman JC, Humphries MJ, Bussolino F and Serini G: Neuropilin-1/GIPC1 signaling regulates alpha5beta1 integrin traffic and function in endothelial cells. PLoS Biol 7: e25, 2009.

22. Rudchenko S, Scanlan M, Kalantarov G, Yavelsky V, Levy C, Estabrook A, Old L, Chan GL, Lobel L and Trakht I: A human monoclonal autoantibody to breast cancer identifies the PDZ domain containing protein GIPC1 as a novel breast cancer-associated antigen. BMC Cancer 8: 248, 2008.
23. Westbrook JA, Cairns DA, Peng J, Speirs V, Hanby AM, Holen I, Wood SL, Ottewell PD, Marshall H, Banks RE, et al: CAPG and GIPC1: Breast cancer biomarkers for bone metastasis development and treatment. J Natl Cancer Inst 108: 2016.

24. Chittenden TW, Pak J, Rubio R, Cheng H, Holton K, Prendergast N, Glinskii V, Cai Y, Culhane A, Bentink S, et al: Therapeutic implications of GIPC1 silencing in cancer. PLoS One 5: e15581, 2010

25. Bae S, Bessho Y, Hojo M and Kageyama R: The bHLH gene Hes6, an inhibitor of Hes1, promotes neuronal differentiation. Development 127: 2933-2943, 2000.

26. Hartman J, Lam EW, Gustafsson JA and Ström A: Hes-6, an inhibitor of Hes-1, is regulated by 17beta-estradiol and promotes breast cancer cell proliferation. Breast Cancer Res 11: R79, 2009.

27. Akhmanova $A$ and Hoogenraad CC: Microtubule minus-end-targeting proteins. Curr Biol 25: R162-R171, 2015.

28. Fu Y, Zheng S, An N, Athanasopoulos T, Popplewell L, Liang A, $\mathrm{Li} \mathrm{K}, \mathrm{Hu} \mathrm{C}$ and $\mathrm{Zhu} \mathrm{Y}$ : $\beta$-catenin as a potential key target for tumor suppression. Int J Cancer 129: 1541-1551, 2011.

29. Castellana B, Escuin D, Pérez-Olabarria M, Vázquez T, Muñoz J, Peiró G, Barnadas A and Lerma E: Genetic up-regulation and overexpression of PLEKHA7 differentiates invasive lobular carcinomas from invasive ductal carcinomas. Hum Pathol 43 1902-1909, 2012.

30. Tanaka N, Meng W, Nagae S and Takeichi M: Nezha/CAMSAP3 and CAMSAP2 cooperate in epithelial-specific organization of noncentrosomal microtubules. Proc Natl Acad Sci USA 109: 20029-20034, 2012

31. Soung YH, Lee JW, Kim SY, Nam SW, Park WS, Lee JY, Yoo NJ and Lee SH: Mutational analysis of the kinase domain of MYLK2 gene in common human cancers. Pathol Res Pract 202: 137-140, 2006

32. Rong Y, Jin D, Hou C, Hu J, Wu W, Ni X, Wang D and Lou W: Proteomics analysis of serum protein profiling in pancreatic cancer patients by DIGE: Up-regulation of mannose-binding lectin 2 and myosin light chain kinase 2. BMC Gastroenterol 10: 68, 2010.

33. Damasco C, Lembo A, Somma MP, Gatti M, Di Cunto F and Provero P: A signature inferred from drosophila mitotic genes predicts survival of breast cancer patients. PLoS One 6: e14737, 2011.

34. Tabernero J, Cervantes A, Rivera F, Martinelli E, Rojo F, von Heydebreck A, Macarulla T, Rodriguez-Braun E, Eugenia Vega-Villegas M, Senger S, et al: Pharmacogenomic and pharmacoproteomic studies of cetuximab in metastatic colorectal cancer: Biomarker analysis of a phase I dose-escalation study. J Clin Oncol 28: 1181-1189, 2010. International (CC BY-NC-ND 4.0) License. 